Aus der Provinzial-Heil- und Pflegeanstalt in Bonn. (Direktion: Geheimrat A. Westphal.)

\title{
Einwirkung der Lumbalpunktion auf das weibliche Genitale?
}

Von`Dr. Eugen Förster.

Die Lumbalpunktion zur Gewinnung ${ }^{\top}$ des Liquor cerebrospinalis ist im Laufe der Zeit ein ausgezeichnetes und unentbehrliches Hilfsmittel der Diagnose geworden. Auch der praktische Arzt wird zuweilen zu ihrer Ausführung gezwungen, wenn sie auch, wie Lewandowsky (Praktische Neurologie furl Aerzte) 
ausführt, in der Privatpraxis ihre großen Bedenklichkeiten wegen der Unannehmlichkeiten und zufälligen Begleiterscheinungen hat. In den Schilderungen dieser letzteren habe ich bei Lewandowsky keinen Hinweis auf eine Einwirkung der Lumbalpunktion auf das weibliche Genitale gefunden; auch in der mir sonst zugänglichen Literatur, einschließlich der geburtshilflich-gynäkologischen, ist die Frage nicht erörtert. ${ }^{1}$ ) Aus diesem Grunde möchte ich einige Beobachtungen mitteilen, die wir in allerjüngster Zeit in der Provinzial-Heil- und Pflegeanstalt Bonn gemacht haben.

Am 14. Mai 1919 wurde die 28 jührige Ehefrau W. in großem Erregungszustande unter dem Bilde der Manie in die Anstalt Bonn eingeliefert. Die Patientin ist im Jahre 1909 wegen eines ahnlichen Zustandes in der Anstalt Teupitz behandelt worden aus der sie nach dreivierteljährigem Aufenthalt als ungeheilt von Dementia paranoides (?) entlassen wurde. (Krankenblatt der Anstalt Teupitz.)

Vor ungefähr Jahresfrist machte sie infolge mißlicher ehelicher Verhältnisse und in pekuniärer Bedrängnis einen Selbstmordversuch.

Bei der Einlieferung in die Anstalt bot Patientin körperlich nichts Auffälliges. Da sie angab (der Ehemann bestätigte es) mehrere Schmierkuren durchgemacht zu haben, ferner während des Krieges nach vier Fehlgeburten einem syphilitischen Mädchen, das jetzt mit Salvarsan behandelt werde, das Leben gegeben zu haben, wurden am 3. Juni vormittags 11 Uhr zur Klärung der Diagnose etwa $12 \mathrm{ccm}$ Liquor cerebrospinalis durch Lumbalpunktion entnommen. (Das Untersuchungsergebnis nach Wa.R. war negativ, auch Nonne war negativ, es bestand keine Zellvermehrung.)

In der auf die Punktion folgenden Nacht abortierte die Patientin. (Sie selbst wußte von der Schwangerschaft nichts, schätzt diese jetzt auf eine etwa zweimonatige.)

Seit dem 24. Januar 1918 befindet sich in der Anstalt die 24 jährige ledige Arbeiterin Martha $\mathrm{B}^{2}$ )

Bei der Einlieferung in die Anstalt war die Patientin, die kurz vorher unehelich geboren hatte, ruhig und geordnet, wohl orientiert und gab freundlich und willig Auskunft. Der $\mathrm{Zu}$ stand änderte sich aber bald. Patientin lag meist im Bett, die Decke über den Kopf gezogen, apathisch, ohne Interesse für ihre Umgebung.

Am 6. April 1918 war sie zum letzten Male menstruiert.

Anfang Juni wurden bei der Patientin typische athetotische Bewegungen in der linken Hand beobachtet, die allmählich auf die rechte Hand und auch auf die beiden unteren Extremitäten abergingen und zu Fixationskontrakturen führten. Erscheinungen von Pro- und Retropulsion traten auf. Starkes Grimassieren stellte sich ein. Von September 1918 ab nahmen die athetotischen Bewegungen immer mehr ab und sind augenblicklich vollständig verschwunden. Zunehmend ist sie auch geistig wieder freier geworden.

Da bei ähnlichen Fällen das Lumbalpunktat positive Wa $\mathrm{R}$. ergeben hat (Moeli), wurde auch bei Patientin die Lumbalpunktion versucht, mißlang aber wegen ihres ungeheuren Sträubens. Ein zweiter Versuch, einige Tage später, am 5. Juni 1918, vormittags $11 \mathrm{Uhr}$, nach einer Skopolamin-Morphiuminjektion, brachte trotz dreimaligen Einstichs keinen Liquor.

In der Nacht nach diesem zweiten Punktionsversuch stellten sich bei der Patientin nach 14 monatiger Pause die Menses wieder ein.

$\mathrm{Ob}$ es sich bei dieser eigenartigen Reaktion vonseiten des weiblichen Genitale bei zwei Patientinnen - bei der einen Abort nach Lumbalpunktion, bei der anderen Wiedereintritt der Menses nach mehrmaligem Einstich - um ein zufälliges Zusammentreffen handelt, wage ich nicht zu entscheiden. Wir haben in der Anstalt bisher auch keine ahnlichen Beobachtungen gemacht, haben sogar eben erst bei einer im siebenten bis achten Monat schwangeren Patientin die Punktion ohne irgendwelche Folgen ausfuhren können.

Es handelt sich um ein 18 jăhriges Dienstmädchen C. ${ }^{3}$ ) Das Mädchen ist unehelich geschwängert worden, und es stellten sich bei ihm Anfang Februar neben Erbrechen eigenartige rhythmische Zuckungen im linken Mundwinkel ein, die sich im Laufe der Zeit auch auf das linke Platysma, den rechten Mundwinkel und den linken Arm und die Finger der linken Hand erstreckten und auch im Schlafe auftraten. Bei dem schwachsinnigen Mädchen, das sich seiner Schwangerschaft garnicht bewußt ist fanden sich bei der körperlichen Untersuchung lebhaft gesteigerte Kniesehnenreflexe, absolut starre Pupillen und allgemeine Hypalgesie. Zur Klärung der Diagnose wurden am 23. Mai 1919 etwa $8 \mathrm{ccm}$ Liquor durch Lumbalpunktion entnommen. Wa.R. negativ.

2) Auch nicht in der Arbeit über die Lumbalpunktion von $\mathrm{K}$ a $\mathrm{r}$ $\mathrm{E} s \mathrm{k}$ u chen, die mir während der Drucklegung dieser Zeilen zu Gesicht kam. - 2) Eine ausführliche Publikation von Geh. Rat W e s tph a l über diesen Fall findet sich im Arch. f. Psych. u. Nervenkrh. 60. H 2/3: Üeber doppeiseitige Athetose und verwandte Krankheitszustände. - 3) Der Fall ist am 16. Juni in der Sitzung der Niederrheinischen Gesellschaft für Natur- und Heilkunde in Bonn von Geh. Rat We s t p hal vorgestellt und ausführlich besprochen worden. Vgl. den Vereinsbericht in dieser Wochenschrift.
Bei dieser Patientin trat, wie schon oben erwähnt wurde keine Reaktion seitens des Genitale ein.

Ich habe die drei Fälle.zusammengestellt, da mir weitere Beobachtungen auf Nebenwirkungen seitens des weiblichen Genitale bei Lumbalpunktion nicht unwichtig erscheinen. Sollten diese in positivem Sinne ausfallen, so scheint es mir Pflicht des Arztes zu sein, sich in allen Fällen vor einer Punktion einigermaßen Gewißheit über bestehende Gravidität, besonders in den ersten Monaten, zu verschaffen. Auch in forensischer Beziehung könnte die angeschnittene Frage zu Bedeutung gelangen, da unter Umstănden eine Abtreibung durch Lumbalpunktion versucht werden könnte. 